\title{
Desarrollo de una Estación Experimental Multipropósito para la Enseñanza en Control de Procesos
}

\author{
Rafael E. Vásquez, Norha L. Posada y Fabio Castrillón \\ Universidad Pontificia Bolivariana, Escuela de Ingenierías, Grupo de Automática y Diseño A+D, Circular 1 \\ \# 70-01, Bloque 11, Medellín-Colombia (e-mail: rafael.vasquez@upb.edu.co).
}

Recibido Dic. 19, 2014; Aceptado Feb. 10, 2015; Versión final Abr. 8, 2015, Publicado Oct. 2015

\begin{abstract}
Resumen
Se presenta el desarrollo de un equipo de laboratorio para el estudio, entrenamiento e investigación en control de procesos. Los requerimientos de diseño fueron determinados por usuarios de diferentes disciplinas relacionados con la academia, la investigación y tareas de entrenamiento a nivel de pregrado, postgrado y consultoría industrial. Se presenta el diseño de un proceso de nivel de líquido de dinámica variable (hasta tercer orden), interactuante, que permite incluir no linealidades y tiempo muerto variable. La estación experimental cuenta con tres opciones tecnológicas para el controlador: PLC, controlador industrial y computador portátil. Esta flexibilidad permite el uso de diversos algoritmos de control: PID, LQG, control difuso, control dinámico matricial y control con modos deslizantes, entre otros. El equipo propuesto cumple con los requisitos educativos incluidos en las tendencias contemporáneas de educación en ingeniería.
\end{abstract}

Palabras clave: control de procesos, educación en ingeniería, equipos de laboratorio, enseñanza del control

\section{Development of a Multipurpose Experimental Station for the Teaching of Process Control}

\begin{abstract}
This paper addresses the development of a lab equipment for studying, training, and research activities in process control. The design requirements were determined by users from several disciplines that perform academic, research and training activities in undergraduate education, graduate studies, and industry consulting. The design process for a level control system, with variable dynamic behavior (up to third order), with interaction, that allows the user to include nonlinearities and variable dead time is presented. The experimental station offers three different technological alternatives for the controller: a PLC, an industrial controller, and a laptop computer. With such flexibility, several control strategies can be implemented: PID, LQG, fuzzy control, dynamic matrix control, and sliding mode control, among others. The proposed lab equipment fulfills educational requirements included in contemporary tendencies of engineering education.
\end{abstract}

Keywords: process control, engineering education, laboratory equipment, lab station, control teaching 


\section{INTRODUCCIÓN}

La educación en ingeniería ha venido cambiando en el mundo, para satisfacer estándares de acreditación, necesidades sociales y necesidades industriales, entre otras (Kadlowec et al., 2007). Existen actualmente diversas tendencias de educación en ingeniería que están basadas en la definición de resultados que se adquieren a lo largo del programa de formación. Por ejemplo, la estrategia propuesta por ABET requiere que cada estudiante complete un proyecto central o curso de diseño (Felder y Brent, 2003). Por otro lado, la estrategia CDIO se concentra en la concepción, diseño, implementación y operación de sistemas reales y productos (Crawley et al., 2007).

En general, las estrategias de educación requeridas por los diferentes sistemas de acreditación buscan que los currículos tengan bases sólidas en ciencias y matemáticas, con la expectativa de éstas se conecten con conceptos relativos a la práctica de la ingeniería. Existen diversos currículos en los cuales se han combinado efectivamente métodos tradicionales de enseñanza con aprendizaje basado en proyectos (Mills y Treagust, 2003). Los laboratorios juegan un papel importante en estos currículos, y Feisel y Rosa (2005) establecieron una lista de objetivos educacionales que deben ser considerados durante la fase de diseño de éstos. El uso de experiencias de laboratorio en control de procesos es importante para la comprensión de conceptos fundamentales y para el fortalecimiento de habilidades que necesitan los profesionales de diversas disciplinas, y que pueden ser adquiridas mediante procesos de aprendizaje activo (Felder y Brent, 2003b). Estas habilidades incluyen, entre otras: solución de problemas, toma de decisiones, trabajo en equipo, habilidades comunicativas (orales y escritas) y pensamiento crítico (Woods et al., 2013).

Los procesos industriales presentan generalmente características que hacen difícil su regulación, por ejemplo: interacciones multivariable entre variables controladas y manipuladas, variables de estado no medibles, perturbaciones no medibles, incertidumbre en los parámetros y variaciones en el tiempo, presencia de tiempo muerto, entre otras (Aström y Hägglund, 2001). Existen diversas opciones que se usan para la implementación de sistemas automáticos de control. Éstas incluyen desde el uso de controladores clásicos, donde el control PID sigue siendo el más utilizado dadas sus características de implementación, robustez frente a perturbaciones y simplicidad matemática (Shinskey, 1994; Luyben y Luyben, 1996; Sung et. al, 1996; Aström y Hägglund, 2001) hasta controladores predictivos basados en modelo como el control dinámico matricial que se ha usado ampliamente durante los últimos 20 años para sistemas multivariables (Ravi et. al, 2012; Darby et. al, 2012).

La educación en control de procesos requiere de la comprensión de conceptos fundamentales y cantidades físicas relacionadas con la dinámica de los procesos (Dormido, 2004). En la literatura se encuentran reportados diversos trabajos relacionados con equipos para enseñanza del control automático, incluyendo sistemas en los cuáles el usuario tiene interacción física o interacción remota con el equipo y sistemas virtuales (Horacek, 2000; Leva, 2006; Keller, 2006; Aliane et al., 2007; Márquez, 2008; Stefanovic et al., 2009; Deschenes et al., 2013; Charre-lbarra et al., 2014; He et al., 2014).

La Universidad Pontificia Bolivariana publicó su Modelo Pedagógico Integrado (UPB, 2009), el cual orienta todos sus programas a nivel de pregrado y postgrado. Este modelo, en el cual se enmarca el desarrollo de la estación experimental, establece la necesidad de una opción curricular integrada que entre otros aspectos promueva el aprendizaje activo y propicie el vínculo entre experiencias de aprendizaje y esquemas de significado. Sumado a lo anterior, Camacho, Celis, Arenas y Duque (2014), afirman que para que se produzca aprendizaje, el sujeto debe encontrarse suficientemente involucrado en la actividad propuesta y ésta debe ser relevante para el aprendizaje que se busca.

Este trabajo presenta el desarrollo de un equipo de laboratorio multipropósito, que puede ser utilizado para educación en control de procesos, a nivel de pregrado, posgrado y entrenamiento industrial, y que adicionalmente, dada su flexibilidad y posibilidad de reconfiguración, es una herramienta útil para investigación en ingeniería de control, tema en el cual tanto la teoría como la práctica son importantes (Rhinehart, 2013). La planta está constituida por un sistema de tanques en serie a escala de laboratorio y dotada con instrumentación industrial robusta que ofrece diversas opciones y condiciones de proceso tales como: dinámica de diferentes órdenes, posibilidad de interacción, tiempo muerto variable, comportamiento lineal, comportamiento no lineal, inclusión de perturbaciones, etc., además de usar diferentes tecnologías para la implementación de controladores, dependiendo del público objetivo que la utiliza.

\section{REQUERIMIENTOS DE DISEÑO}

La estación experimental se diseñó para cumplir con los requerimientos de usuario expresados por docentes que imparten cursos en diferentes niveles de formación: pregrado, postgrado y entrenamiento industrial, e investigadores de diversas disciplinas tales como: ingeniería química, ingeniería de procesos, ingeniería 
mecánica, ingeniería de control e instrumentación, ingeniería eléctrica/electrónica, entre otras. Los requerimientos que fueron determinados para un equipo multipropósito son: 1) Proceso a escala que tenga instrumentación industrial para el control y manipulación de variables; 2) Medición de variables controladas, manipuladas y perturbaciones; 3) Diferentes configuraciones para la dinámica del sistema: primer orden, segundo orden, tercer orden; comportamiento lineal y no lineal; interacción entre variables y tiempo muerto; 4) Uso de diferentes tecnologías de control; 5) Posibilidad de implementar diversas estrategias de control; 6) Posibilidad crear acceso remoto; 7) Uso de materiales que permitan directa visualización de las variables de proceso; y 8) Tamaño compacto para reducir el uso de espacio en laboratorio.

Adicionalmente, para el diseño de la estación experimental desde el punto de vista pedagógico, se plantearon los siguientes resultados de aprendizaje, cuyas bondades y características son presentadas por Spurlin et al. (2008): a) Identificar componentes y acciones básicas de un sistema automático de control, utilizando normas internacionales; b) Describir las características de señales y protocolos de comunicación, e implementar sistemas de adquisición de datos; c) Caracterizar y manipular diferentes elementos de medición usados en procesos industriales; d) Obtener el modelo matemático de un proceso mediante pruebas en planta; e) Diferenciar entre las acciones proporcional, integral y derivativa en controladores PID; f) Usar diferentes algoritmos y tecnologías de control para un proceso real; h) Usar y comparar diferentes métodos de sintonía de controladores utilizando índices de desempeño y robustez; i) Identificar los aspectos más relevantes para la selección, manufactura, operación y mantenimiento de elementos que hacen parte de un sistema automático de control. El mapeo detallado entre los resultados de aprendizaje y las características técnicas de diseño mencionadas anteriormente está fuera de alcance de este trabajo.

\section{DISEÑO Y FABRICACIÓN DE LA ESTACIÓN EXPERIMENTAL}

El diseño de la estación experimental se realizó en tres etapas: diseño conceptual, diseño básico y diseño de detalle.

\section{Diseño conceptual}

Existen diversas opciones de sistemas que permiten satisfacer los requerimientos de usuario. Sin embargo, los sistemas de tanques han sido reportados en la literatura como una opción interesante dadas sus posibilidades de reconfiguración y de visualización de variables (Horacek, 2000). Usando la información provista por los potenciales usuarios se generó, a partir de una lluvia de ideas, un boceto a mano alzada del diagrama simplificado de instrumentación y proceso (P\&ID) el cual contiene componentes básicos. Esto corresponde al diseño conceptual de la estación experimental, Figura 1.

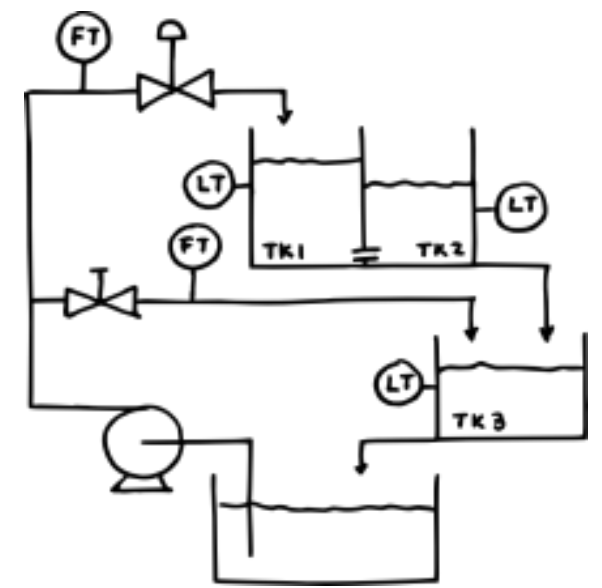

Fig. 1: Boceto del diagrama de instrumentación y proceso (P\&ID)

El proceso está conformado por un tanque superior, mostrado en la Figura 1 como dos tanques interactuantes (Tk1 y Tk2), y el tanque inferior (Tk3). Se muestra la opción de medición de nivel en cada tanque con transmisores denotados como LT. Una bomba lleva el fluido (agua) de un reservorio a dos ramas diferentes en las cuales se indica medición de flujo con transmisores denotados como FT. El ramal principal de tubería cuenta con una válvula de control, la cual permite manipulación del flujo, y el lazo secundario muestra una válvula manual que permite introducir perturbaciones. El tanque superior tiene una división removible, permitiendo al usuario seleccionar un tanque grande, un tanque pequeño o dos tanques interactuantes. Con este concepto, la dinámica del sistema puede seleccionarse según lo indicado en la Tabla 1. 
El comportamiento lineal o no lineal del sistema se introduce con el uso de vertederos de pared delgada, con diferentes características, ubicados en la salida de los tanques Tk2 y Tk3. Entre las opciones de vertederos se encuentran: en $\mathrm{V}$, rectangular, lineal, etc. El tiempo muerto es creado con la adición de una tubería removible en la descarga del tanque Tk2; esta tubería puede seleccionarse lo suficientemente larga para crear un tiempo muerto variable, lo cual representa un reto interesante para el control, cuando el tiempo muerto se aproxima a la constante de tiempo dominante del proceso.

Tabla 1: Posibles configuraciones de la estación experimental

\begin{tabular}{|l|c|c|}
\hline \multicolumn{1}{|c|}{ Combinación de tanques } & Orden & Interacción \\
\hline Un tanque grande (Tk1+Tk2) & 1 & No \\
\hline Un tanque pequeño (Tk2) & 1 & No \\
\hline Dos tanques grandes en serie (Tk1+Tk2), (Tk3) & 2 & No \\
\hline Un tanque pequeño (Tk2) en serie con un tanque grande (Tk3) & 2 & $\mathrm{No}$ \\
\hline Dos tanques pequeños interactuantes (Tk1), (Tk2) & 2 & $\mathrm{Si}$ \\
\hline $\begin{array}{l}\text { Dos tanques pequeños interactuantes (Tk1), (Tk2) en serie con un } \\
\text { tanque grande (Tk3) }\end{array}$ & 3 & $\mathrm{Si}$ \\
\hline
\end{tabular}

\section{Diseño básico}

En la etapa de diseño básico se seleccionaron los componentes requeridos para el sistema, incluyendo la instrumentación que comprende elementos para la medición de variables y los actuadores. La instrumentación del sistema se lista en la Tabla 2. Adicionalmente, se evaluaron diferentes tecnologías de control con el fin de satisfacer los requisitos de flexibilidad para la implementación de diversas estrategias de control, uso de diferentes controladores y posibilidades de acceso remoto en trabajos futuros. Dado que la estación experimental se usará para labores de docencia, investigación y entrenamiento industrial, se estableció el uso de tres tecnologías, seleccionables por el usuario a la hora de controlar el proceso: controlador lógico programable, controlador industrial, control por computador

Controlador Lógico Programable (PLC): Siemens SIMATIC S7-1200. Este es un elemento robusto que permite manejar las señales del proceso a través de módulos de entradas y salidas análogas y digitales. Puede ser conectado fácilmente a otros dispositivos a través de Ethernet y podrá ser usado directamente con una interfaz hombre-máquina (HMI). Este controlador es normalmente usado en procesos industriales y tiene módulos PID integrados, y permitirá el desarrollo de acceso remoto en trabajos futuros.

Controlador industrial: Foxboro 762CNA. Este tipo de controlador es usado comúnmente en la industria de procesos, por lo tanto es una opción indispensable como herramienta para el entrenamiento industrial. Es una opción interesante para procesos de sintonía de controladores PID, tiene opción de implementación de controladores de adelanto/atraso, compensación de tiempo muerto y pre-filtro en la entrada. Este controlador se conecta con el PLC usando las entradas y salidas análogas.

Control por computador: computador portátil con LabVIEW®. Esta tecnología permite la implementación de todo tipo de algoritmos de control: clásicos, por acción pre-calculada, controladores inteligentes, controladores no lineales y observadores de estado, entre otros. Adicionalmente, presenta la opción de guardar datos directamente en el computador para ser procesados fuera de línea. El computador se conecta con el PLC usando Ethernet.

Tabla 2: Instrumentación de la estación experimental

\begin{tabular}{|l|l|l|l|}
\hline \multicolumn{1}{|c|}{ Elemento } & \multicolumn{1}{c|}{ Marca } & \multicolumn{1}{c|}{ Modelo } & \multicolumn{1}{c|}{ Características técnicas } \\
\hline Medidores de nivel & Siemens & SITRANS P DSIII & $\begin{array}{l}\text { Rango 1-60 mbar, salida 4-20 mA, protocolo Hart e } \\
\text { indicación local }\end{array}$ \\
\hline Medidores de flujo & Siemens & $\begin{array}{l}\text { SITRANS FM } \\
\text { MAG5100W }\end{array}$ & Rango 0-10 m/s, salida 4-20 mA e indicación local \\
\hline Válvula de control & Spirax-Sarco & LEA31 & $\begin{array}{l}\text { Carrera } 20 \mathrm{~mm} \text {, coeficiente de flujo de 7.4 } \\
\text { gpm/psi } 1 / 2\end{array}$ \\
\hline Posicionador/convertidor & Spirax-Sarco & PN9120E & Entrada 4-20 mA y salida 3-15 psi \\
\hline Bomba & Pedrollo & CP620D & Rango 0-6 $\mathrm{m}^{\wedge} 3 / \mathrm{h}$ \\
\hline Variador de frecuencia & Yaskawa & AC Drive-J1000 & Alimentación 110 V AC \\
\hline Compresor & KCT & 431-2525 & Capacidad de 7 cfm \\
\hline
\end{tabular}




\section{Diseño de detalle}

El diseño detallado de la estación requirió de diferentes cálculos y selección de componentes, y se dividió en tres áreas: componentes mecánicos, componentes eléctricos y componentes computacionales. Se usó un proceso iterativo de diseño hasta obtener un sistema que satisface los requerimientos de diseño establecidos por los usuarios.

\section{Componentes mecánicos:}

i) Dimensionamiento de tanques: se realizó teniendo en cuenta el rango de los transmisores de presión diferencial usados para la medición de nivel y el máximo flujo dado por la bomba. Se seleccionó acrílico transparente para su fabricación dado que este material permite visualización directa de las variables. El tanque reservorio se dimensionó con el máximo volumen de líquido que circula por el sistema y el nivel mínimo requerido en la succión de la bomba.

ii) Dimensionamiento de vertederos: se dimensionaron cuatro vertederos, uno lineal y tres no lineales, para ser usados en la descarga de los tanques, Figura 2a.

iii) Tiempo muerto: se dimensionó una tubería larga que reemplaza uno de los vertederos en el momento en el que se quiera crear un retardo de transporte para el flujo que va del tanque superior al tanque inferior, Figura 2b.

iv) Elementos estructurales: para soportar el sistema se diseñó una estructura de aluminio, la cual es reconfigurable en caso de necesitar aditamentos futuros. El diseño de las tuberías se hizo con un software de modelación tridimensional, con el fin de verificar interferencias y problemas de manufactura.

\section{Componentes eléctricos:}

i) Fuente de potencia: se dimensionó la fuente del sistema para cubrir el consumo de potencia de los elementos involucrados en la estación, teniendo en cuenta requerimientos de voltaje y corriente para alimentación.

ii) Actuación de la bomba: el motor de la bomba es de velocidad variable, por lo tanto se seleccionó un variador electrónico de frecuencia Yaskawa J1000, el cual permite ajustar la velocidad del rotor y generar diferentes condiciones de operación de la planta o ser usado como elemento final de control.

iii) Panel frontal: se diseñó para contener la pantalla táctil del PLC (HMI), los botones de arranque y paro, paro de emergencia, el controlador industrial y el variador de velocidad de la bomba.

iv) Gabinete eléctrico: se integró en la parte superior de la estructura. Dos lados del gabinete se construyeron en acrílico transparente para permitir la visualización interna de las conexiones y distribución de los componentes eléctricos. El gabinete cuenta con las protecciones eléctricas adecuadas para garantizar la seguridad de la operación y de los usuarios.
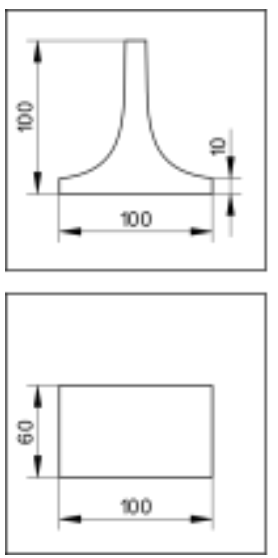

(a)
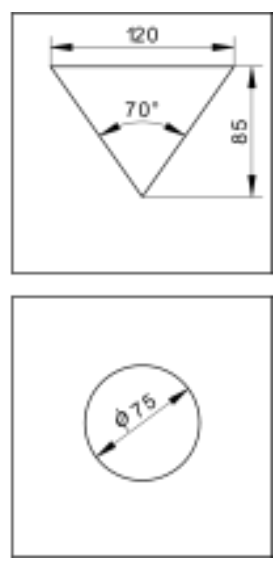

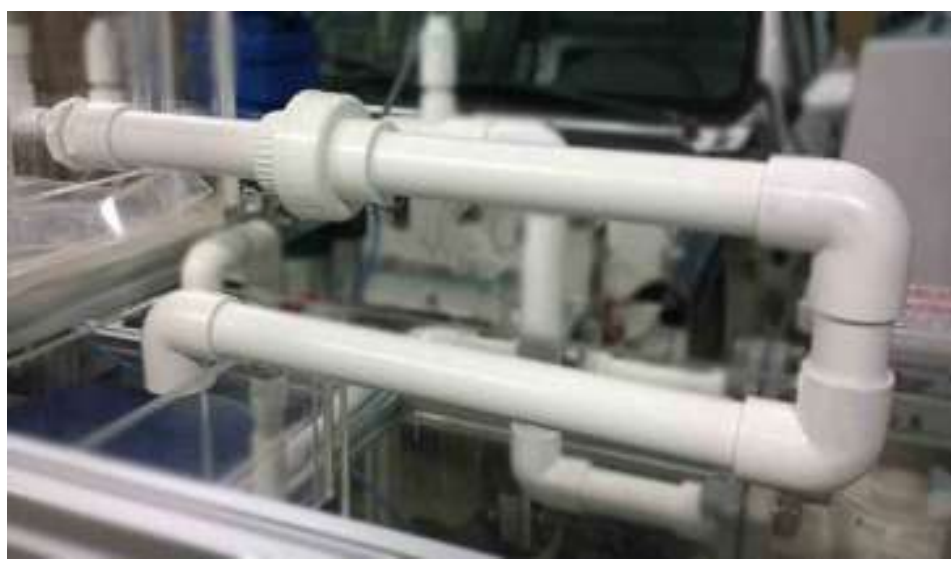

(b)

Fig. 2: Componentes mecánicos. (a) Vertederos de pared delgada. (b) Tubería para tiempo muerto. 


\section{Componentes computacionales:}

i) Aplicación del PLC: se desarrolló una aplicación para adquisición y manejo de señales dado que el computador lógico programable es el componente que permite recibir las señales de los sensores y enviar las señales a los actuadores. Adicionalmente, el PLC cuenta con aplicaciones para implementación de controladores, cuando se desea realizar control con esta opción tecnológica.

ii) Interfaz de LabVIEW: se requiere para la comunicación entre el computador portátil y el PLC. Adicionalmente se pueden usar diversos algoritmos implementados como funciones, cuando se selecciona esta tecnología para el control del proceso.

iii) Administración de datos: se desarrolló para permitir que los usuarios puedan exportar los datos y almacenarlos en formato de texto plano con el fin de realizar procesamiento fuera de línea.

La Figura 3a muestra el prototipo virtual del sistema obtenido al final del proceso de diseño. Todos los componentes industriales fueron obtenidos a través de proveedores locales para la industria de procesos, y la manufactura de todos los componentes adicionales requeridos se hizo en talleres locales. El ensamble se hizo en los laboratorios de la universidad, Figura 3b. La Figura 4 muestra el diagrama de instrumentación y proceso (P\&ID) simplificado, basado en la norma ANSI/ISA-5.1-2009 (ISA, 2009).

\section{RESULTADOS}

La estación experimental fue diseñada, en principio, para acompañar procesos de aprendizaje a nivel de pregrado y posgrado, por lo tanto se espera que los estudiantes que interactúan con el nuevo equipo tengan experiencias que les permitan concebir, diseñar, implementar y operar sistemas automáticos de control. La flexibilidad dada al equipo permite realizar diversas actividades que conducen a cumplir los diferentes objetivos de aprendizaje planteados en la sección de los requerimientos de diseño, entre éstas se encuentran: identificación de componentes, modelación de sistemas dinámicos, variación de parámetros, cambios en condiciones y puntos de operación, diseño e implementación de controladores, análisis de robustez, etc.

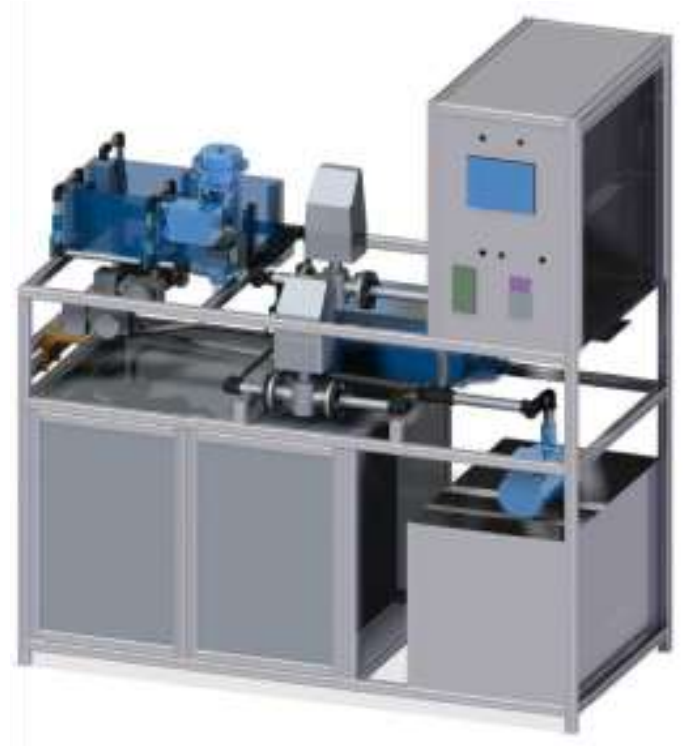

(a)

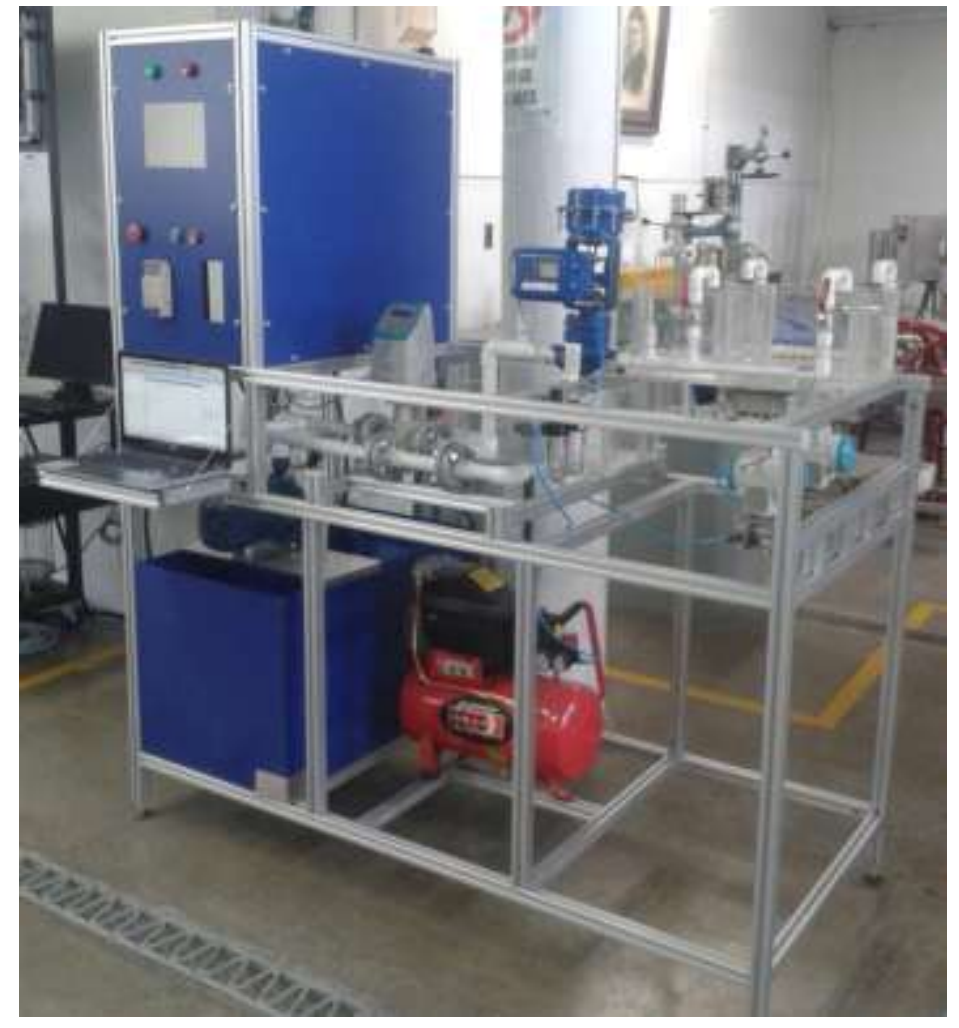

(b)

Fig. 3: Diseño y construcción. (a) Prototipo virtual. (b) Proceso de ensamble del equipo.

\section{Prácticas de laboratorio para control de procesos}

A continuación se proponen algunas posibles sesiones de laboratorio para cursos de control de procesos en el cual puede ser usada la estación experimental: 
1. Introducción: acciones básicas de los sistemas automáticos de control.

2. Identificación de componentes usando la norma ANSI/ISA-5.1-2009 (ISA, 2009).

3. Señales y protocolos de comunicación.

4. Sistemas de adquisición de datos.

5. Instrumentos de medición, transmisores y convertidores.

6. Válvulas de control: selección y caracterización en planta.

7. Identificación de sistemas dinámicos con pruebas en planta.

8. Tecnologías de control: PLC, controladores industriales y control por computador.

9. Diseño de sistemas de control asistido por computador (CACSD).

10. Control PID: acciones proporcional, integral y derivativa.

11. Sintonía de controladores PID.

12. Control moderno: control por retroalimentación de estado y observadores de estado.

13. Comparación de estrategias avanzadas de control: control de matriz dinámica (DMC) vs. control con modos deslizantes (SMC).

14. Selección de componentes para la implementación de sistemas de control.

15. Seguridad y confiabilidad en sistemas de control.

\section{Lazos de control y modelo matemático}

Para evidenciar algunos tipos de prácticas que se pueden realizar con el equipo, se presentan a continuación algunos ejemplos relativos a las acciones básicas de un sistema de control, a la identificación de componentes usando la norma ANSI/ISA-5.1-2009 (ISA, 2009), modelización matemática e identificación de sistemas de con pruebas en planta.

\section{Acciones básicas e identificación de componentes}

La Figura 4 muestra que en el sistema desarrollado se tienen múltiples lazos de control. Por ejemplo:

1. Control retroalimentado de nivel en el tanque Tk3: a) Sensor transmisor (LT 100-03); b) Adquisición de datos y manejo de señales (URU 100-01); c) Controlador (URU 100-01 ó UIRC 100-01 ó LIC 100-01); d) Elemento final de control (UV 100-01).

2. Control retroalimentado de flujo en ramal de tubería superior: a) Sensor transmisor (FIT 100-01); b) Adquisición de datos y manejo de señales (URU 100-01); c) Controlador (URU 100-01 ó UIRC 100-01); d) Elemento final de control (UV 100-01 ó UIY 100-01).

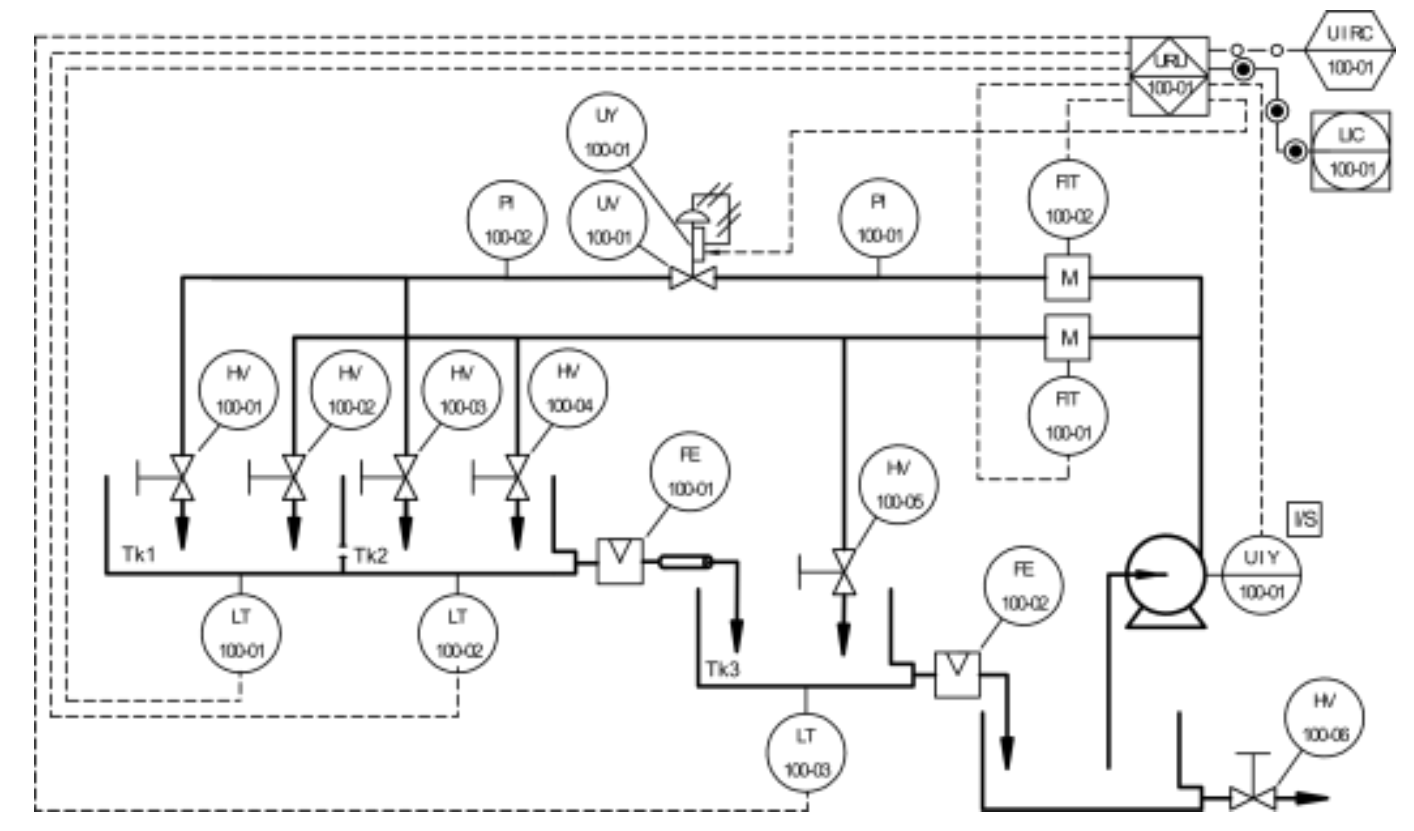

Fig. 4: Diagrama de instrumentación y proceso (P\&ID).

Otros lazos retroalimentados adicionales se pueden seleccionar teniendo en cuenta las tres acciones básicas de un sistema de control: medición (algún sensor transmisor de nivel o flujo), decisión (alguno de los tres controladores) y acción (alguno de los dos actuadores); la Tabla 1 puede ayudar en el proceso de selección. 


\section{Modelo matemático para el sistema de dos tanques en serie no interactuantes}

En este caso se presenta un ejemplo de modelización para la tercera opción presentada en la Tabla 1 (Tk1+Tk2, Tk3); la variable controlada es el nivel del tanque Tk2. Las ecuaciones que representan la dinámica se pueden obtener con un balance de masa en cada uno de los tanques, dado por

$$
\dot{m}_{i}(t)-\dot{m}_{o}(t)=\frac{d m(t)}{d t},
$$

donde $\dot{m}_{i}(t)$ y $\dot{m}_{o}(t)$ son los flujos másicos de entrada y salida, respectivamente, y el lado derecho representa la variación de la masa de líquido en el tiempo. Dado que el fluido de trabajo es agua a condiciones estándar $\left(25^{\circ} \mathrm{C}\right.$ y una presión absoluta de $\left.100 \mathrm{kPa}\right)$, se considera que la densidad es constante y (1) se convierte en

$$
q_{i}(t)-q_{o}(t)=A \frac{d h(t)}{d t},
$$

donde $A$ es el área del tanque, $q_{i}(t)$ y $q_{o}(t)$ son los flujos volumétricos de entrada y salida, respectivamente, y el lado derecho representa la variación del nivel de líquido en el tiempo. A partir de (2) se puede escribir el conjunto de ecuaciones diferenciales para los dos tanques no interactuantes de la forma

$$
\begin{aligned}
& A_{12} \dot{h}_{12}(t)+q_{2}\left(h_{12}\right)=q_{i}(t), \\
& A_{3} \dot{h}_{3}(t)+q_{3}\left(h_{3}\right)=q_{2}\left(h_{12}\right),
\end{aligned}
$$

donde $A_{12}$ es el área del tanque superior, $A_{3}$ es el área del tanque inferior, $q_{\mathrm{i}}(\mathrm{t})$ es el flujo de entrada al tanque superior, $q_{2}\left(h_{12}\right)$ es el flujo de salida del tanque superior y $q_{3}\left(h_{3}\right)$ es el flujo de salida del tanque inferior. Las funciones que representan los flujos de salida de los dos tanques en (3) y (4), dependen del vertedero de pared delgada seleccionado en cada caso, y se muestran en la Tabla 3.

\section{Tabla 3: Ecuaciones para los vertederos de pared delgada}

\begin{tabular}{|l|l|l|}
\hline \multicolumn{1}{|c|}{ Tipo de vertedero } & \multicolumn{1}{|c|}{ Linealidad } & \multicolumn{1}{c|}{ Ecuación } \\
\hline Lineal & Lineal & $q(h)=K L \sqrt{a}\left(h+\frac{5 a}{8}\right)$ \\
\hline En V & No lineal & $q(h)=1.4 h^{5 / 2}$ \\
\hline Rectangular & No lineal & $q(h)=1.838\left(L-\frac{2 h_{1}}{10}\right) h^{3 / 2}$ \\
\hline Circular & No lineal & $q(h)=1.518 D^{0.693} h^{1.807}$ \\
\hline
\end{tabular}

\section{Identificación del sistema con una prueba en planta}

Una de las actividades sugeridas para ser realizadas en la estación experimental es la identificación de sistemas dinámicos. Para este propósito se hizo un experimento sencillo con la planta en modo de control manual, con vertederos lineales en la salida de ambos tanques (superior Tk1+Tk2 e inferior Tk3), se hizo un cambio tipo escalón en la apertura de la válvula de control (de 30\% a 40\%) y se registró la variación de la altura en el tanque Tk3, Figura 5. Se utilizó el paquete de identificación de sistemas de Matlab®, y con los datos de entrada y salida del sistema se obtuvo una aproximación lineal en la zona de operación dada por

$$
G(s)=\frac{0.522}{55.3 s^{2}+14.9 s+1}[=] \frac{\%}{\%},
$$

donde las unidades de la ganancia de la función de transferencia en están dadas en porcentaje de salida de transmisor sobre porcentaje de apertura de la válvula de control. La Figura 5 muestra la respuesta en el tiempo del sistema de segundo orden sobre amortiguado ante una entrada tipo escalón, dado por (5), superpuesta con los datos obtenidos para el nivel en el tanque Tk3 en la prueba experimental. Este modelo lineal se ajusta al modelo teórico que se obtendría usando aproximaciones lineales para (3) y (4). 
(a)

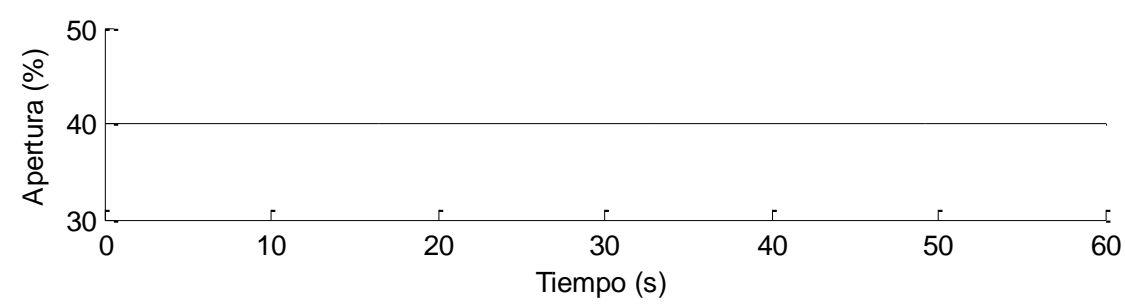

(b)

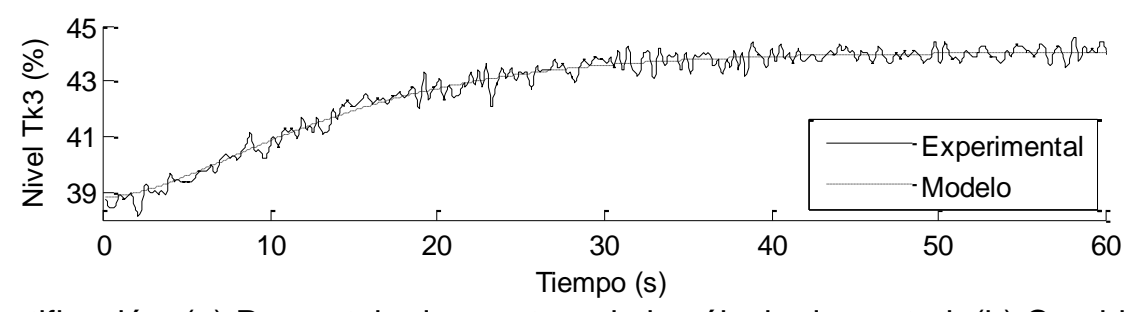

Fig. 5: Prueba de identificación. (a) Porcentaje de apertura de la válvula de control. (b) Cambio del nivel en Tk3 y respuesta en el tiempo para el nivel con la función de transferencia obtenida.

\section{CONCLUSIONES}

Se presentó el desarrollo de un equipo de laboratorio multipropósito, utilizado para la educación de fundamentos en sistemas dinámicos y control de procesos, con una orientación dada a satisfacer requisitos educacionales propuestos en modelos de acreditación internacionales tales como ABET y CDIO. Adicionalmente, el equipo está pensado para ser utilizado en actividades de entrenamiento industrial y experimentación de sistemas avanzados de control dentro de proyectos de investigación en teoría de control.

La arquitectura propuesta cuenta con elementos industriales, y diversas opciones para monitoreo y control, lo que da la posibilidad no sólo de prestar servicios educativos formales a nivel de pregrado y posgrado, sino que brinda la posibilidad de crear escenarios para entrenamiento industrial tanto con presencia física en el laboratorio, como con acceso remoto que podrá ser implementado en una etapa futura.

Dadas las características de la estación experimental, se tiene la posibilidad de contar diversos sistemas dinámicos, desde lineales de primer orden hasta no lineales de tercer orden interactuantes con tiempo muerto variable. Esto abre la posibilidad al uso de estrategias como PID, PID con autosintonía, control dinámico matricial (DMC) y control con modos deslizantes (SMC), lógica difusa, redes neuronales, algoritmos genéticos, etc.

La estación desarrollada permite crear prácticas de laboratorio en diversos temas del control de procesos, que promuevan el aprendizaje activo y propicien el vínculo entre experiencias de aprendizaje y esquemas de significado, según lo establecido en el Modelo Pedagógico Integrado de la UPB. Estas experiencias deben ser diseñadas para que se cumplan los resultados de aprendizaje declarados y usados como insumo en la etapa de diseño del equipo.

\section{AGRADECIMIENTOS}

El presente trabajo se desarrolló con la financiación de los laboratorios de las facultades de Ingeniería Química e Ingeniería Mecánica y del Centro de Investigación para el Desarrollo y la Innovación - CIDI, pertenecientes a la Universidad Pontificia Bolivariana, Sede Central Medellín, dentro del proyecto "Estudio comparativo de estrategias avanzadas de control en un sistema de tanques", radicado 025B-02/13-22.

\section{REFERENCIAS}

Aliane, N., J. Fernández, A. Martínez y J. Ortiz, Un Laboratorio de Ingeniería de Control Basado en Internet, doi: 10.4067/S0718-07642007000600004, Información Tecnológica: 18(6), 19-26 (2007)

Aström, K. y T. Hägglund, The Future of PID Control, doi: 10.1016/S0967-0661(01)00062-4, Control Engineering Practice: 9(11), 1163-1175 (2001)

Camacho, A., A. Arenas, J. Celis y M. Duque, Dime cómo enseñas y te diré que tanto aprenden los estudiantes, ACOFI, Bogotá, Colombia (2014)

Charre-lbarra, S.M., J.A. Alcalá-Rodríguez, N. López-Luiz y M. A. Durán-Fonseca, Sistema Didáctico de Control de Presión, doi: 10.4067/S0718-50062014000500005, Formación Universitaria: 7(5), 33-40 (2014) 
Crawley, E. F., J. Malmqvist, J., S. Östlund y D.R. Brodeur, Rethinking Engineering Education. The CDIO Approach, doi: 10.1007/978-0-387-38290-6, Springer, New York, USA (2007)

Darby, M.L. y M. Nikolaou, MPC: Current Practice and Challenges, doi: 10.1016/j.conengprac.2011.12.004, Control Engineering Practice: 20(4), 328-342 (2012)

Deschenes, J.S., N. Barka, M. Michaud, D. Paradis y J. Brousseau, A Joint Learning Activity in Process Control and Distance Collaboration Between Future Engineers and Technicians, Chemical Engineering Education: 47(1), 9-14 (2013)

Dormido, S., Control Learning: Present and Future, doi: 10.1016/j.arcontrol.2003.12.002, Annual Reviews in Control: 28(1), pp. 115-136 (2004)

Feisel, L.D. y A.J. Rosa, The Role of the Laboratory in Undergraduate Engineering Education, doi: 10.1002/j.2168-9830.2005.tb00833.x, Journal of Engineering Education, 94, 121-130 (2005)

Felder, R.M. y R. Brent, Designing and Teaching Courses to Satisfy the ABET Engineering Criteria, doi: 10.1002/j.2168-9830.2003.tb00734.x, Journal of Engineering Education: 92(1), 7-25 (2003)

Felder, R.M. y R. Brent, Learning by Doing, Chemical Engineering Education: 37(4), 282-283 (2003)

$\mathrm{He}, \mathrm{Z}$., Z. Shen y S. Zhu, Design and Implementation of an Internet-Based Electrical Engineering Laboratory, doi: 10.1016/j.isatra.2013.12.035, ISA Transactions: 53(5), 1377-1382 (2014)

Horacek, P., Laboratory Experiments for Control Theory Courses: A survey, doi: 10.1016/S13675788(00)90029-4, Annual Reviews in Control: 24, pp. 151-162 (2000)

ISA, ANSI/ISA-5.1-2009 Instrumentation Symbols and Identification Standard, International Society of Automation (2009)

Kadlowec, J., K. Bhatia, T.R. Chandrupatla, J.C. Chen, E. Constans, H. Hartman, A.J. Marchese, P. von Lockette, y $\mathrm{H}$. Zhang, Design Integrated in the Mechanical Engineering Curriculum: Assessment of the Engineering Clinics, doi: 10.1115/1.2722788, Journal of Mechanical Design: 129(7), 682-691 (2007)

Keller, J., Interactive Control System Design, doi: 10.1016/j.conengprac.2004.12.014, Control Engineering Practice: 14(2), 177-184 (2006)

Leva, A., A Simple and Flexible Experimental Laboratory for Automatic Control Courses, doi: 10.1016/j.conengprac.2004.10.011, Control Engineering Practice: 14(2), 167-176 (2006)

Luyben, M. y W. Luyben, Essentials of Process Control, McGraw-Hill (1996)

Márquez, D.A. y O.O. Cárdenas, Implementación de un Laboratorio Virtual para la Enseñanza de Controladores PID, doi: 10.1612/inf.tecnol.3884it.07, Información Tecnológica: 19(3), pp. 75-78 (2008)

Mills, J.E., y D.F. Treagust, Engineering Education - Is Problem-Based or Project-Based Learning the Answer?, Australasian Journal of Engineering Education: 3, pp. 2-16 (2003)

Ravi, V.R., T. Thyagarajan y G.U. Maheshwaran, Dynamic Matrix Control of a Two Conical Tank Interacting Level System, doi: 10.1016/j.proeng.2012.06.306, Procedia Engineering: 38, 2601-2610 (2012)

Rhinehart, R.R., ISA Transactions 50th Year Recognition - and Some Perspectives, doi: 10.1016/j.isatra.2012.11.003, ISA Transactions: 52(1), pp. 2- 5 (2013)

Shinskey, F., Feedback Controllers for the Process Industries, McGraw-Hill, Singapore (1994)

Spurlin, J., S. Rajala y J. Lavelle. Designing better engineering education through assessment, Stylus publishing, USA (2008)

Sung, S.W. y I.B. Lee, Limitations and Countermeasures of PID Controllers, doi: 10.1021/ie960090+, Industrial \& Engineering Chemistry Research: 35(8), pp. 2596-2610 (1996)

Stefanovic, M., V. Cvijetkovic, M. Matijevic y V. Simic, A LabVIEW-Based Remote Laboratory Experiments for Control Engineering Education, doi: 10.1002/cae.20334, Computer Applications in Engineering Education: 19(3), 538-549 (2011)

UPB - Universidad Pontificia Bolivariana, Modelo pedagógico integrado (2009), http://www.upb.edu.co/pls/portal/docs/PAGE/GP_REPOSITORIO_IMAGENES/PG_RIMG_MEDELLIN/MOD ELO\%20PEDAGOGICO.PDF. Acceso: 1 de Abril (2015)

Woods, D.R., D. Briedis y A. Perna, Professional Skills Needed by Our Graduates, Chemical Engineering Education: 41(2), 81-90 (2013) 